\title{
Implementing Innovative Gaze Analytic Methods in Clinical Psychology
}

\author{
A Study on Eye Movements in Antisocial Violent Offenders
}

\author{
Nina A. Gehrer \\ Michael Schönenberg* \\ Clinical Psychology \& Psychotherapy \\ University of Tübingen \\ [nina.gehrer,michael.schoenenberg] \\ @uni-tuebingen.de
}

\author{
Andrew T. Duchowski ${ }^{\dagger}$ \\ Visual Computing \\ Clemson University \\ duchowski@clemson.edu
}

\author{
Krzysztof Krejtz \\ Psychology Department \\ SWPS University of Social Sciences \& \\ Humanities \\ kkrejtz@swps.edu.pl
}

\begin{abstract}
A variety of psychological disorders like antisocial personality disorder have been linked to impairments in facial emotion recognition. Exploring eye movements during categorization of emotional faces is a promising approach with the potential to reveal possible differences in cognitive processes underlying these deficits. Based on this premise we investigated whether antisocial violent offenders exhibit different scan patterns compared to a matched healthy control group while categorizing emotional faces. Group differences were analyzed in terms of attention to the eyes, extent of exploration behavior and structure of switching patterns between Areas of Interest. While we were not able to show clear group differences, the present study is one of the first that demonstrates the feasibility and utility of incorporating recently developed eye movement metrics such as gaze transition entropy into clinical psychology.
\end{abstract}

\section{CCS CONCEPTS}

- Applied computing $\rightarrow$ Psychology;

\section{KEYWORDS}

eye tracking, antisocial offenders, facial emotion recognition

\section{ACM Reference format:}

Nina A. Gehrer, Michael Schönenberg, Andrew T. Duchowski, and Krzysztof Krejtz. 2018. Implementing Innovative Gaze Analytic Methods in Clinical Psychology. In Proceedings of ETRA '18: 2018 Symposium on Eye Tracking Research \& Applications, Warsaw, Poland, June 14-17, 2018 (ETRA'18), 9 pages. https://doi.org/10.1145/3204493.3204543

\footnotetext{
${ }^{*}$ This study was funded by the German Research Foundation (Scho 1448/3-1).

${ }^{\dagger}$ This work was supported by the U.S. National Science Foundation (grant IIS-1748380).
}

Permission to make digital or hard copies of all or part of this work for personal or classroom use is granted without fee provided that copies are not made or distributed for profit or commercial advantage and that copies bear this notice and the full citation on the first page. Copyrights for components of this work owned by others than ACM must be honored. Abstracting with credit is permitted. To copy otherwise, or republish, to post on servers or to redistribute to lists, requires prior specific permission and/or a fee. Request permissions from permissions@acm.org.

ETRA'18, June 14-17, 2018, Warsaw, Poland

(C) 2018 Association for Computing Machinery.

ACM ISBN 978-1-4503-5706-7/18/06 ..\$15.00

https://doi.org/10.1145/3204493.3204543

\section{INTRODUCTION}

The ability to decode nonverbal social information in order to infer the emotional state of an interaction partner is crucial for effective social interaction. Accordingly, individuals are able to quickly and efficiently identify emotional expressions from specific facial cues [Smith et al. 2005; Tracy and Robins 2008]. These cues are similar across cultures, at least for the six basic emotions, i.e., anger, disgust, fear, happiness, sadness, and surprise [Ekman 1999; Ekman and Friesen 1971]. The accurate interpretation of emotional expressions is based on the processing of relevant regions of the face and directing visual attention to them (e.g., wide-open fearful eyes or smiling happy mouth) [Eisenbarth and Alpers 2011; Schurgin et al. 2014]. Thus, tracking eye movements while viewing emotional faces is a promising approach to gain insight into the processes underlying categorization of emotions.

In clinical research, eye tracking can be a useful tool to explore deviations in scanning patterns that could account for emotion recognition impairments associated with psychological disorders. Impairments in facial affect recognition have been linked to the development and maintenance of various psychological disorders including autism [Uljarevic and Hamilton 2013], depression [Dalili et al. 2015], anxiety disorders [Demenescu et al. 2010], schizophrenia [Kohler et al. 2009], attention-deficit hyperactivity disorder [Bora and Pantelis 2016], and antisocial personality disorder (ASPD) and psychopathy [Dawel et al. 2012; Marsh and Blair 2008].

The majority of clinical studies exploring eye movements while viewing faces does not tap the potential of the myriad analytical methods available. Although analysis of dwell time or number of fixations to certain Areas of Interest (AOIs) can yield interesting findings, an inclusion of more innovative and complex analytical methods (e.g., sequential analysis of eye movements) may add valuable information. Here, we present an analysis of scan patterns while viewing faces including widely-used standard eye movement parameters (e.g., total dwell time) as well as more recently developed metrics such as gaze transition entropy [Krejtz et al. 2015]. Based on these measures, we investigate group differences in attention orienting to the eyes, extent of exploration behavior and structure of switching patterns between AOIs in antisocial violent offenders (AVOs) and a matched healthy control group. 


\section{BACKGROUND}

We start by outlining the motives for exploring scan patterns of AVOs while categorizing emotional faces in the present study. After introducing the clinical constructs of ASPD and psychopathy and the associated emotion recognition deficit, we present a possible mechanism that might underlie these impairments, i.e., deficient attentional orienting to the eyes. Based on a review of previous studies investigating the relation between attention to the eyes and psychopathic traits, we describe the design of the current study and introduce our selection of eye movement parameters.

\subsection{Emotion Recognition: ASPD \& Psychopathy}

A large proportion of incarcerated offenders fulfill the diagnostic criteria for ASPD (e.g., up to $72.7 \%$ in Germany) [Kopp et al. 2011] ASPD is characterized by a pervasive pattern of disregard for and violation of the rights of others and evolves from early behavioral tendencies before the age of 15 years [American Psychiatric Association 2000]. The clinical construct of psychopathy describes a similar psychopathology characterized by antisocial, irresponsible, and impulsive behavior but emphasizes interpersonal and affective abnormalities (e.g., superficial charm, callousness/lack of empathy) [Hare 2003]. Thus, psychopathy is more narrowly defined and most psychopaths fulfill the criteria for ASPD [American Psychiatric Association 2000] but not vice versa. Consistently, the prevalence rate of psychopathy in prison is about 15\% [Hare 2003].

According to the violence inhibition mechanism model, a basic impairment in decoding social signals of distress is assumed to play a critical role in the etiology of antisocial behavior [Blair 1995, 2001]. In healthy individuals emotional distress cues (e.g., sad or fearful facial expressions) usually elicit empathy and inhibit aggressive behavior. This mechanism is crucial for socialization and the development of morality. An insensitivity to these social cues in antisocial and psychopathic populations could thus contribute to the development and maintenance of aggressive psychopathology.

Accordingly, deficits in emotion recognition, e.g., in the recognition of fearful faces, have been documented in ASPD and psychopathy [Dawel et al. 2012; Marsh and Blair 2008; Wilson et al. 2011]. A recent systematic review confirmed an impaired facial affect recognition in violent offenders in general, including marked deficits in decoding of fear, disgust, and anger [Chapman et al. 2017].

\subsection{Attention to the Eyes \& Psychopathic Traits}

Adolphs et al. [2005] proposed that fear recognition deficit can be caused by a general lack of spontaneous fixations on the eyes when they investigated a patient with bilateral amygdala lesion. The amygdala is a subcortical brain structure that has repeatedly been linked to the processing of fear and the widened fearful eyes in particular [Morris et al. 1996; Whalen et al. 2004]. Since the wide open eyes are a crucial feature of a fearful expression [Smith et al. 2005; Whalen et al. 2004], instructing the patient to look at the eyes of the facial stimuli leads to a temporal correction of the recognition deficit. Subsequent study findings corroborated a role of the amygdala in the quick detection of and orientation of attention to emotionally salient facial features like the eyes [Gamer and Büchel 2009; Gamer et al. 2013]. An altered amygdala response to fearful expressions has been linked to aggressive behavior and psychopathy [Jones et al. 2009; Lozier et al. 2014; Marsh et al. 2008].

Dadds et al. [2008, 2006] investigated gaze patterns to facial emotional stimuli in a sample of healthy boys with high and low callous-unemotional $(\mathrm{CU})$ personality traits. These traits reflect the affective core component of psychopathy. Consistently, high CU traits were associated with less fixations on the eye region and a lower accuracy in fear recognition. Again, an instruction to attend to the eyes reversed this impairment. Two recent studies investigated scan patterns while viewing emotional faces in healthy adults and showed a link between higher psychopathic traits and reduced attention to the eyes [Boll and Gamer 2016; Gillespie et al. 2015]. Boll and Gamer reported the analysis of number of saccades and latency of the first saccade as measures of intensity of face scanning and general vigilance towards facial cues. In their sample, psychopathic traits were inversely related to these measures indicating that deviations of eye movement patterns in psychopaths might not be constrained to reduced attention to the eyes.

To date, only two clinical groups have been studied. A group of adolescents with conduct disorder (precursor of ASPD before the age of 18) showed reduced attention to the eyes for sad and fearful faces and a lower accuracy for emotion recognition [Martin-Key et al. 2017]. In contrast, a group of adult violent offenders did not differ from controls in terms of attention to the eyes or recognition performance [Gillespie et al. 2017]. However, psychopathic traits were inversely associated with attention to the eyes specifically for surprise in adolescents and across all emotions in violent offenders.

There is evidence for an impairment in directing attention to the eyes of emotional faces in individuals with high psychopathic traits, which may due to deficient functioning of the amygdala and is assumed to underlie the deficit in facial affect recognition. However, eye movements were related to differing aspects of psychopathy (affective/interpersonal factor of psychopathy vs. antisocial behavior vs. boldness) and only two studies reported significant but weak associations between attention to the eyes and accuracy of emotion recognition [Gillespie et al. 2015; Martin-Key et al. 2017].

\subsection{Current Study}

The aim of the current study was to explore gaze patterns in a group of male AVOs and a matched healthy control group. In two tasks, participants were asked to either categorize the gender of facial affective stimuli or their emotional expression, as scan patterns may vary according to the nature of the categorization task [Schyns et al. 2002; Smith and Merlusca 2014]. Therefore, the additional gender discrimination task served as a control task to explore viewing patterns independent of task demands [Adolphs et al. 2005; Scheller et al. 2012]. Further, there is evidence that the start position of gaze at stimulus onset influences subsequent eye movements: Arizpe et al. [2012] argue that a central fixation cross allows a more detailed processing of the fixated stimulus area prior to the initial saccade and thus biases the position of the first fixation. Thus, we presented a fixation cross on either side of the screen prior to stimulus onset.

In the first step of the analysis, we examined two different measures of attention to the eyes. Total dwell time was calculated as a commonly used general index of attention during the entire stimulus presentation. This variable was assumed to be influenced by 


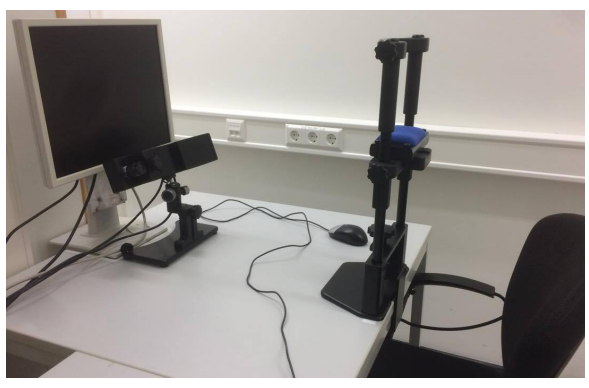

(a) setup

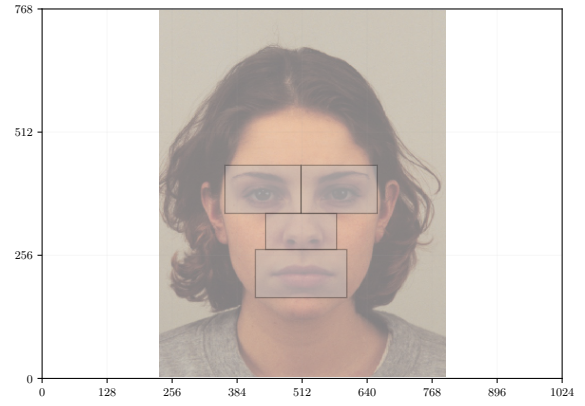

(b) AOI definition

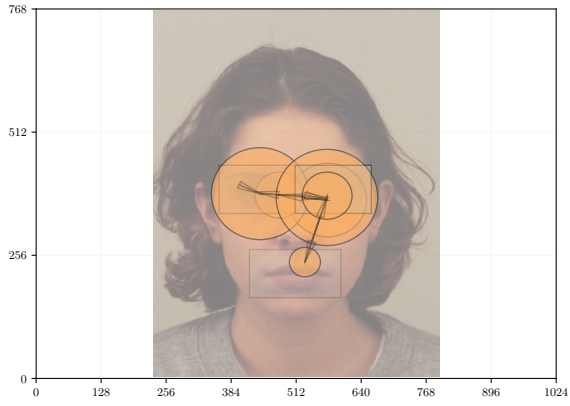

(c) scanpath

Figure 1: Experimental setup (a) and example of a neutral face image with a depiction of defined Areas Of Interest (AOIs) used in the analysis (b) and a typical recorded scanpath visualization composed of fixations connected by saccades (c).

top-down processes (e.g., task) and bottom-up (stimulus-driven) factors. Further, we analyzed the location of the first fixation on the image after stimulus onset and calculated the frequency of this initial fixation on the eye region as a measure for spontaneous attention orienting to the eyes. This parameter might be more strongly influenced by bottom-up (e.g., salient fearful widened eyes or a happy smile) than by top-down factors because initial saccades have been shown to be less susceptible to voluntary control/goaldirected mechanisms than later eye movements [Nummenmaa et al. 2006]. We predicted the AVO group to show reduced attention to the eyes compared to controls across both tasks and all emotions (derived from Adolphs et al. [2005]). This was hypothesized to be indicated by dwell time and especially frequency of the initial fixation on the eye region since impaired bottom-up processes were shown to be the reason for the lack of spontaneous fixations to the eyes in a patient with bilateral amygdala lesion [Kennedy and Adolphs 2011]. Additionally, we expected to find a relation between reduced attention to the eyes and high psychopathic traits.

Since there is evidence that attention orienting mechanisms may be more generally impaired in association with high psychopathic traits [Dawel et al. 2015], we included two additional metrics in our analysis. First, we calculated the number of gaze transitions between the important diagnostic regions of the faces i.e., left and right eye, nose, and mouth. Gaze transitions between these diagnostic features should vary depending on how much information is needed to be processed and evaluated for the categorization decision. Second, we investigated the structure of the viewing patterns by calculating gaze transition entropy [Krejtz et al. 2015]. This parameter was developed to allow comparisons of the predictability of fixation transitions between AOIs and thus can be an index of how structured vs. chaotic the switching patterns are. Investigation of group differences and correlations with psychopathic traits using both of these innovative metrics is explorative and may be useful in developing new hypotheses about altered scanning patterns underlying emotion recognition deficit in antisocial populations.

\section{METHODOLOGY}

In the present study, we collected eye movement data while categorizing gender and emotional expressions of displayed faces in
AVOs and a healthy control group. Clinical interviews, self-report questionnaires, and an intelligence test were used to assess characteristics of all participants.

\subsection{Participants}

Twenty-one antisocial male offenders convicted for violent crimes were recruited from a cooperating German correctional facility (Justizvollzugsanstalt Rottenburg). All fulfilled the following inclusion criteria: Between 18 and 65 years of age, sufficient knowledge of the (German) language and no self-reported current or history of psychotic symptoms. Three of the AVOs had to be excluded due to technical problems during recording of the eye movement data resulting in a final sample of $n=18$. They had been charged with sentences ranging from 7 months to lifetime for crimes such as (aggravated) assault $(\mathrm{n}=7),($ attempted) murder $(\mathrm{n}=4)$, (attempted) manslaughter $(n=3)$, (aggravated) robbery $(n=2)$, or (attempted) extortion under threat of force $(n=2)$.

Healthy male individuals with no history of criminal offenses $(n=21)$ were recruited from the institute's database and via social media (e.g., facebook posts). All participants gave written informed consent and received monetary compensation for participation. The study was approved by the local ethics committee and was conducted in accordance with the Declaration of Helsinki.

\subsection{Diagnostic Measures}

Current or lifetime psychiatric disorders were assessed with the Mini International Neuropsychiatric Interview (MINI) [Sheehan et al. 2010] according to DSM-IV criteria (Diagnostic and Statistical Manual of Mental Disorders) [American Psychiatric Association 2000]. Psychopathic personality traits and aggressive behavior were measured using two self-report questionnaires. The 64item Self-Report Psychopathy scale (SRP-III) [Paulhus et al. 2009] yields a total score and scores for the four factors of psychopathy: Interpersonal Manipulation, Callous Affect, Erratic Lifestyle, and Antisocial Behavior. The 29-item Buss-Perry Aggression Questionnaire (BPAQ) [Buss and Perry 1992; Herzberg 2003] assesses aggression on four subscales, i.e., Physical Aggression, Verbal Aggression, Anger, and Hostility, which are aggregated to a total score. To measure intelligence, we used the 18 -item nonverbal Wiener 
Matrizen-Test 2 (WMT-2) [Formann et al. 2011]. The WMT-2 is a short version of the original Wiener Matrizen-Test [Formann and Piswanger 1979] that assesses deductive reasoning.

\subsection{Stimuli}

We used images of faces displaying seven different emotional expressions (i.e., angry, disgusted, happy, fearful, neutral, sad, or surprised) with $562 \times 762$ pixel resolution (see Figure $1 \mathrm{~b}$ for a neutral example and presentation size relative to screen). We chose faces of 16 models from the Karolinska Directed Emotional Faces picture set [Lundqvist et al. 1998] following the selection of Eisenbarth and Alpers [2011] who studied gaze patterns associated with different emotional expressions in a previous study. Each model displayed each of the seven emotional expressions yielding a total of 112 images. The 112 images were split into two stimulus sets (four male and four female models each) of comparable recognition rate according to Goeleven et al. [2008]. For each participant, each stimulus set was assigned to either the gender discrimination or the emotional categorization task. Image set assignment to task was balanced to control for possible stimulus effects.

\subsection{Procedure}

The study was designed as a $2($ group $) \times 2($ task $) \times 7$ (emotion $)$ mixed design. Participants were asked to categorize presented emotional faces in two tasks (i.e., gender discrimination and emotion categorization) while their eye movements were recorded. Prior to testing, we conducted a 9-point calibration (average calibration error lower than $0.5^{\circ}$ visual angle). During the recording, data quality was continuously monitored by the experimenter and a drift check was performed at the beginning of each trial (i.e., participants were asked to look at a fixation spot to allow comparison of actual and calculated gaze position). Order of tasks was fixed beginning with the gender discrimination task and followed by the categorization of emotional expressions. Both tasks started with seven practice trials. Next, facial affective stimuli of the assigned set (see § 3.3 for detailed description) were presented with one repetition and in random order resulting in 112 experiment trials per task (i.e., 224 in total). At the beginning of each trial, a fixation cross was displayed either on the left or on the right side of the screen (approximately at the height of the middle between eyes and mouth of the faces). The stimulus was presented for $2500 \mathrm{~ms}$ only after a fixation of $300 \mathrm{~ms}$ was recorded in order to ensured that the current fixation was not within the facial image at stimulus onset. Participants responded during presentation of a response display by clicking on the gender or emotion category. Subsequently, a clinical interview (MINI) was conducted and participants completed the self-report questionnaires and the WMT-2 assessment of deductive reasoning.

\subsection{Apparatus}

Stimuli were displayed on a 19-inch computer screen with $1024 \times 768$ pixel resolution. Each participant's chin was placed on a chin rest to minimize head movement and to ensure a viewing distance of $60 \mathrm{~cm}$ (see Figure 1a). Eye movements were recorded binocularly at a sampling rate of $500 \mathrm{~Hz}$ using an SR Research EyeLink 1000 eye tracker. ${ }^{1}$ As display computer we used an HP laptop controlling

\footnotetext{
${ }^{1}$ http://www.sr-research.com
}

stimulus presentation and data collection via Experiment Builder software from SR Research [2008].

\subsection{Eye Movement Data Analysis}

Analysis of gaze patterns was based on four predefined Areas of Interest (AOIs), i.e., left eye, right eye, nose, and mouth (see Figure 1b). For analyzing attention to the eyes, the AOIs for the left and right eye were combined. After sample output reports were created with SR Research's Data Viewer, data were further processed using Duchowski's [2017] Gaze Analytics Pipeline (implemented in Python), consisting of the following (customized) steps:

(1) denoise and extract raw gaze data $g_{i}=\left(x_{i}, y_{i}, t_{i}\right)$ from the vendor's exported files, where $\left(x_{i}, y_{i}\right)$ coordinates indicate the position of the gaze point, and $t_{i}$ indicates the timestamp,

(2) filter raw gaze data to detect fixations $f_{i}=\left(x_{i}, y_{i}, t_{i}, d_{i}\right)$, where $\left(x_{i}, y_{i}\right)$ now indicate the centroid of the fixation, $d_{i}$ the fixation's duration, and $t_{i}$ the timestamp as before,

(3) collate fixation-related data for statistical analysis.

In the present case, following Siegenthaler et al. [2014], the denoising step included removal of data $200 \mathrm{~ms}$ before the start of, and $200 \mathrm{~ms}$ following the end of a blink, as identified by the eye tracker (other methods are also available, e.g., see Jiang et al. [2013]). In the filtering step, data was converted to visual angle given screen resolution (1024 $\times 768$ pixels), diagonal dimensions (19-inch), and assumed viewing distance $(60 \mathrm{~cm})$. A 7-tap Savitzky-Golay [1964] filter was then used to differentiate positional gaze data to produce velocity. The Savitzky-Golay filter was set to use a $3^{\text {rd }}$ degree polynomial to fit the data (see Gorry [1990] for further filter usage details). Saccade detection was based on a velocity threshold of $50^{\circ}$ per second.

Subsequently, dependent eye movement variables were separately calculated for each participant and for each task and emotion in R [R Core Team 2016]. We analyzed main effects and possible interactions of the factors group, task, and emotion for each eye movement parameter using mixed analysis of variance (ANOVA). Significant interactions were followed by posthoc ANOVAs or adjusted posthoc t tests. Additionally, we tested for associations between eye movement parameters and psychopathic and aggressive personality traits by calculating correlations.

\section{RESULTS}

We first present demographic and clinical characteristics of AVOs and healthy controls, and then report results for recognition performance in both tasks. Subsequently, we provide results of the analysis of eye movement parameters introduced above (§ 2.3).

\subsection{Participant Characteristics}

Demographic and clinical data of offenders and healthy controls are described in Table 1. Both groups did not differ in terms of age, education, or IQ. The AVO group reported significantly higher levels of psychopathic and aggressive traits.

Eight AVOs reported a history of substance or alcohol abuse or addiction and five reported symptoms of a previous major depression. Only one participant in the healthy control group reported a history of substance abuse. 

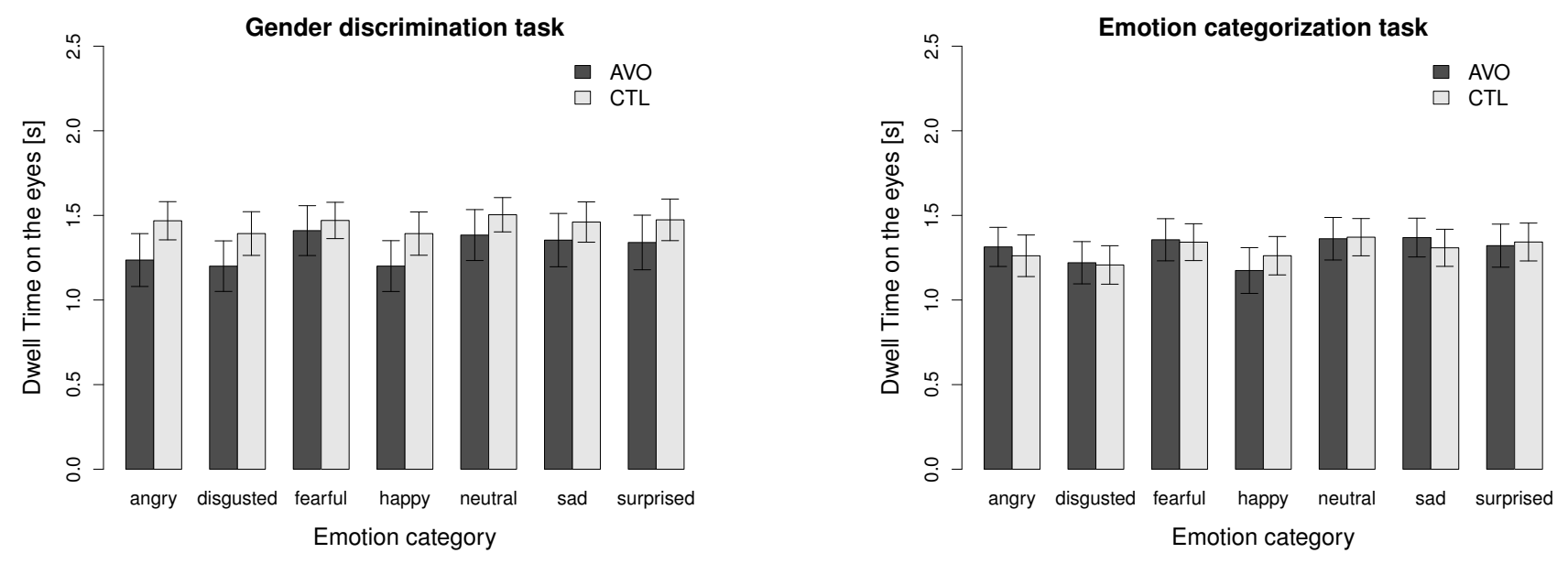

(a) total dwell time on the eyes
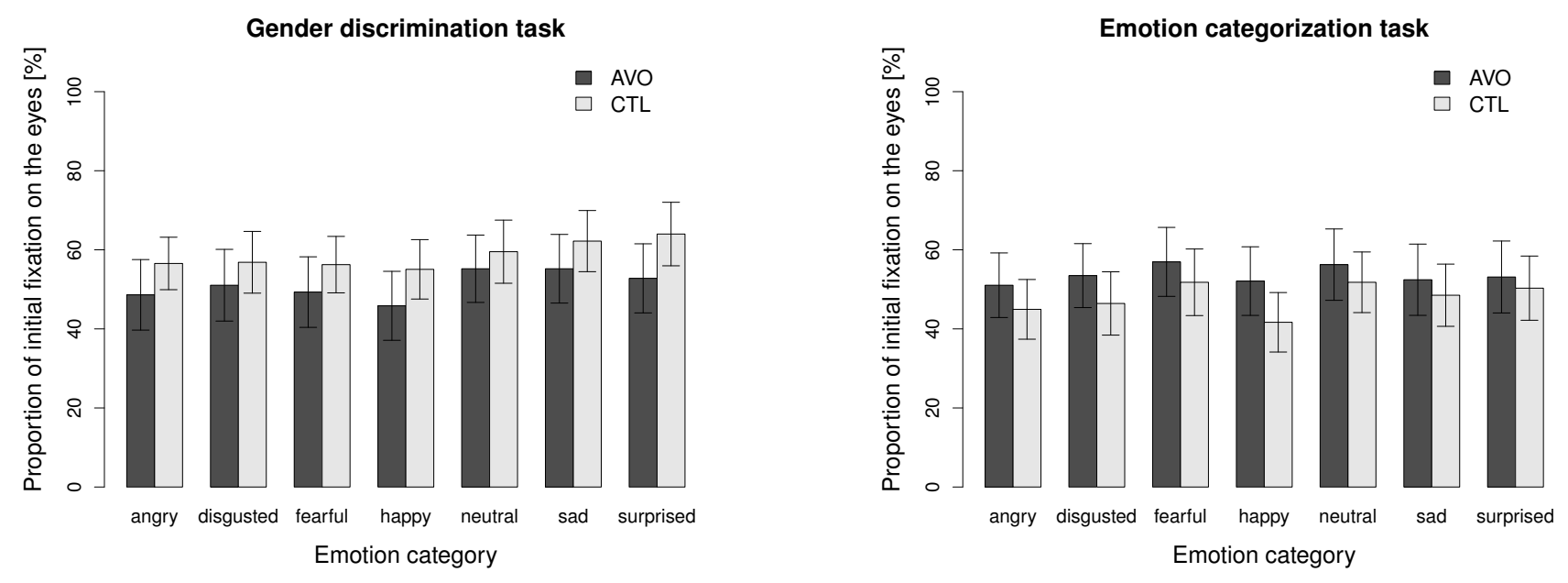

(b) frequency of initial fixation on the eyes

Figure 2: Eye movement parameters describing attention to the eyes for both groups (AVO, antisocial violent offender group; CTL, control group), both tasks, and emotion categories.

\subsection{Behavioral Data}

AVOs and healthy controls performed equally well on the gender discrimination task. The overall accuracy was high $(99.45 \%$ correct). However, categorizing emotions was generally more difficult (80.33\% correct). Both groups showed a comparable performance in this task (AVO group: $\mathrm{M}=81.34 \%, \mathrm{SD}=24.77$; control group: $M=79.17 \%, S D=22.23$ ). Consistent with findings of previous studies (see e.g., Gillespie et al. [2017, 2015]), recognition of fearful faces was least accurate (43.59\% correct) whereas accuracy was best for happy faces $(97.28 \%$ correct).

\subsection{Eye Tracking Data}

Prior to analysis of dynamic eye movements, we calculated the ratio of fixation count within the AOIs and total number of fixations. The proportion of fixations within AOIs was high for antisocial offenders $(M=93.30 \%, S D=5.27)$ and for healthy controls $(M=$ $93.96 \%, \mathrm{SD}=3.37)$ with no differences between groups, $t(28.01)=$ $0.45, p=.653$. The results were comparable to previous findings (see e.g., Schurgin et al. [2014]). In this way, we verified the definition of AOIs and ensured that all frequently fixated regions were included in the analysis.

4.3.1 Attention to the Eyes. Figure 2 shows the results for the eye movement parameters measuring attention to the eye region. For dwell time (see Figure 2a), there was no main effect of group, $F(1,37)=0.19, p=.667$, and no significant two- or three-way interaction, all $F<2.07$, all $p>.130$, indicating that, in general, attention to the eye region was similar between antisocial offenders and healthy controls. The main effect of task was non-significant, $F(1,37)=2.13, p=.153$. Only the displayed emotion significantly influenced the time spent fixating the eyes, $F(1,37)=16.47, p<.001$, 

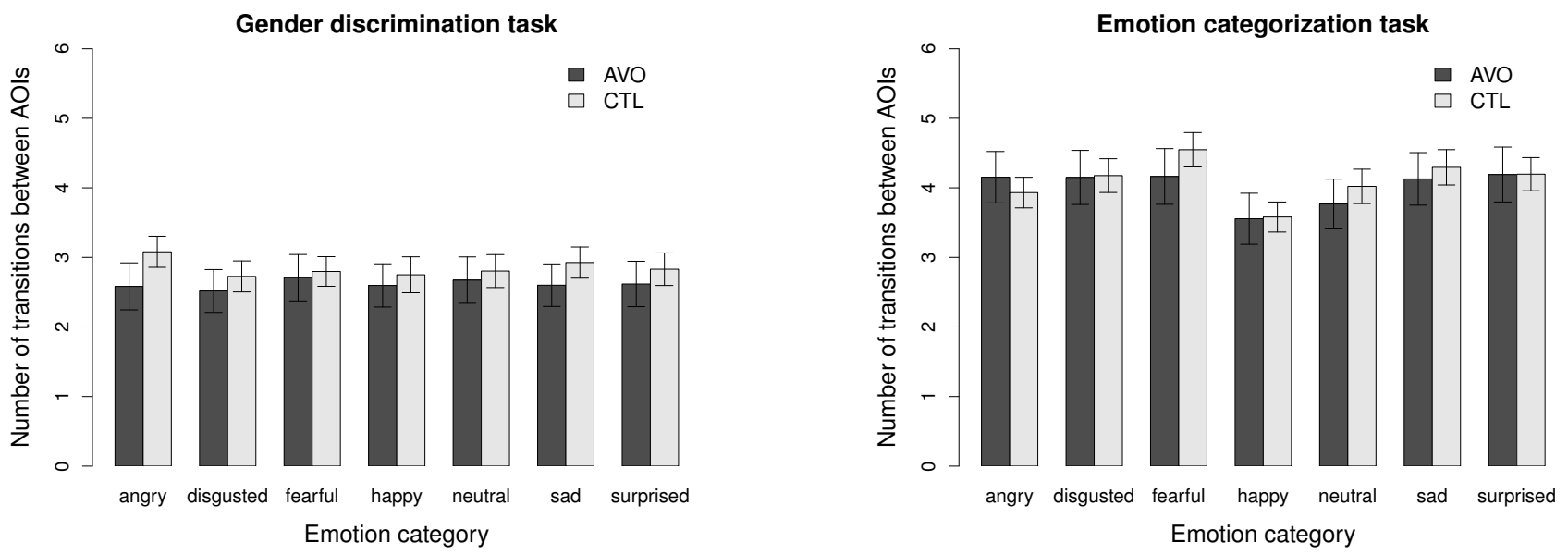

Figure 3: Number of transitions between the AOIs for both groups (AVO, antisocial violent offender group; CTL, control group), both tasks, and emotion categories.

with disgusted and happy expressions drawing the shortest average dwell time on the eyes compared to the other emotions, all $p<.023$ (except from happy compared to angry faces, $p=.182$ ). Fearful and neutral expressions evoked the longest dwell times on the eyes but were only significantly higher than for disgusted, happy and angry faces, all $p<.008$.

For the frequency of the initial fixation on the eye region (see Figure $2 \mathrm{~b})$, the main effect for group was non-significant, $F(1,37)=$ $0.01, p=.935$. However, there was a significant interaction between group and task, $F(1,37)=5.12, p=.030$. Follow-up ANOVAs for both groups separately indicated an effect of task only for the control

Table 1: Demographic and clinical sample characteristics.

\begin{tabular}{lccl}
\hline Variable & AVO $(\mathrm{n}=18)$ & CTL $(\mathrm{n}=21)$ & $\mathrm{t}(37)$ \\
\hline Age & $34.00(9.56)$ & $34.10(11.18)$ & 0.03 \\
Education (years) & $9.27(1.63)$ & $9.90(1.14)$ & 1.37 \\
WMT-2 Sum Score & $7.06(2.98)$ & $8.57(3.49)$ & 1.46 \\
SRP & & & \\
$\quad$ Total Score & $2.79(0.40)$ & $2.22(0.39)$ & $4.54^{* * *}$ \\
$\quad$ Interpers. Manip. & $2.52(0.35)$ & $2.27(0.42)$ & $2.04^{*}$ \\
$\quad$ Callous Affect & $2.47(0.51)$ & $2.26(0.40)$ & 1.44 \\
Erratic Lifestyle & $3.13(0.56)$ & $2.60(0.49)$ & $3.09^{* *}$ \\
$\quad$ Antisocial Behavior & $3.03(0.61)$ & $1.74(0.63)$ & $6.48^{* * *}$ \\
BPAQ & & & \\
$\quad$ Total Score & $80.11(18.74)$ & $56.67(15.94)$ & $4.17^{* * *}$ \\
Physical Aggression & $25.44(8.40)$ & $15.95(6.76)$ & $3.85^{* * *}$ \\
Verbal Aggression & $16.56(2.66)$ & $13.10(3.48)$ & $3.51^{* *}$ \\
Anger & $15.61(4.42)$ & $11.38(3.88)$ & $3.15^{* *}$ \\
Hostility & $22.50(6.84)$ & $16.24(5.98)$ & $4.17^{* * *}$ \\
\hline
\end{tabular}

Note. AVO, antisocial violent offender group; CTL, healthy control group; WMT-2, Wiener Matrizen-Test; SRP, Self-Report Psychopathy scale; BPAQ, Buss-Perry Aggression Questionnaire; The data presented in the table refers to means and standard deviations for each measure (in parentheses). ${ }^{* * *} \mathrm{p}<0.001,{ }^{* *} \mathrm{p}<0.01,{ }^{*} \mathrm{p}<0.05$. group, $F(1,20)=7.93, p=.011$, but not for the antisocial offenders, $F(1,17)=0.31, p=.586$. Hence, control participants tended to initially fixate the eyes more often during gender discrimination than during emotion categorization, whereas the task was irrelevant for violent offenders. Consistent with the results for dwell time, the main effect for emotion was significant, $F(1,37)=5.95, p<.001$. However, the influence of emotion was dependent on task which was indicated by a significant interaction between emotion and task, $F(5.42,200.67)=2.31, p=.040$ rendering the influence of the displayed emotional expression more complex.

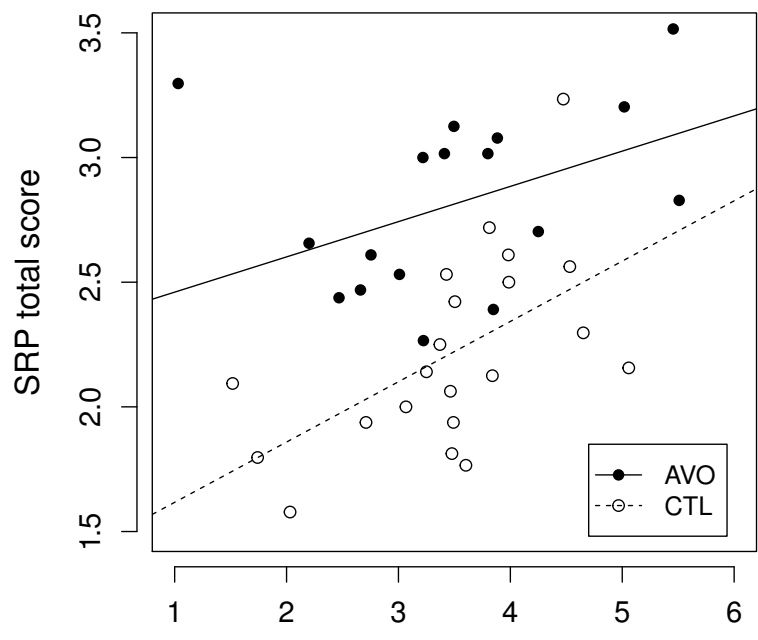

Number of transitions between AOls

Figure 4: Relation of the total score on the Self-Report Psychopathy scale (SRP) and number of transitions between the AOIs across tasks and emotions for both groups (AVO, antisocial violent offender group; CTL, control group). 


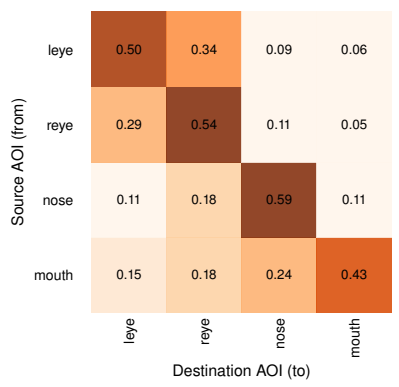

(a) AVO group: gender task

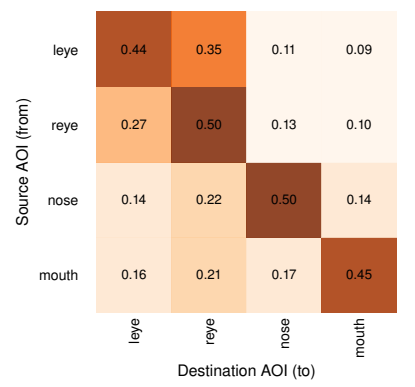

(b) AVO group: emotion task

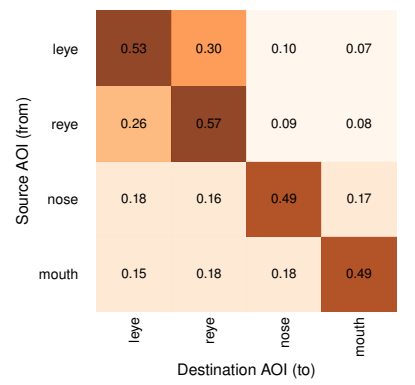

(c) control group: gender task

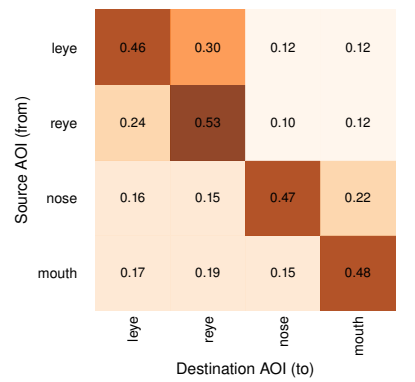

(d) control group: emotion task

Figure 5: Transition matrices of antisocial violent offenders (AVOs) and control group (CTL) for both tasks across emotions. Higher observed probabilities along matrix diagonals indicate a tendency to refixate current AOIs. Higher observed probabilities in the upper-left matrix quadrant are evidence of gaze switching between the two eye regions.

For both reported measures of attention to the eyes, there were no significant correlations with total scores or subscales of selfreported psychopathic or aggressive personality trait measures, all $|r|<.21$, all $p>.189$.

4.3.2 Number of Transitions between AOIs. Figure 3 shows the number of transitions between AOIs, indicating the extent of visual exploration demonstrated by the participants. Analyses yielded no significant effect of group, $F(1,37)=0.19, p=.659$, and no significant two-way interaction with this factor, all $F<0.43$, all $p>.649$. The main effect of task was significant, $F(1,37)=76.85$, $p<.001$, indicating that participants exhibited more AOI transitions during emotion categorization when compared to gender
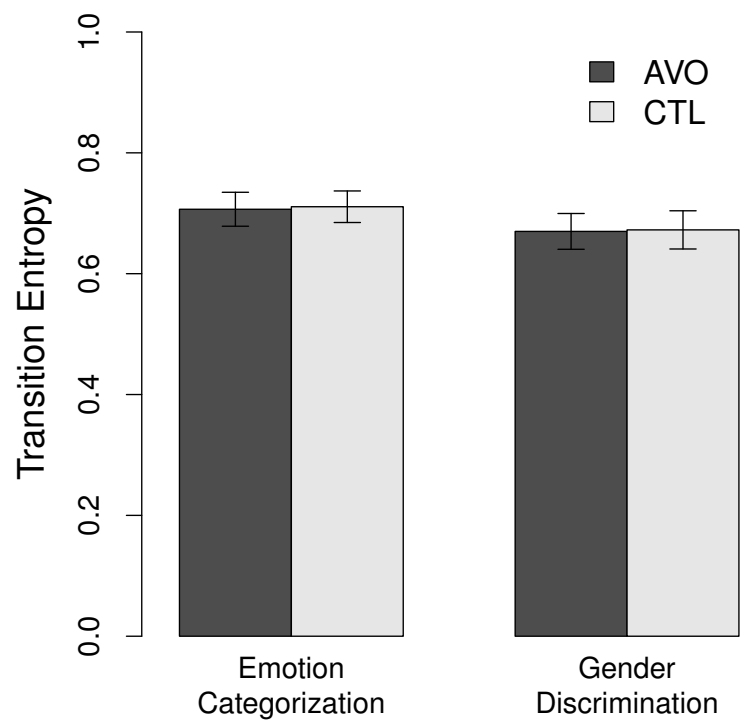

Task

Figure 6: Transition entropy for both groups (AVO, antisocial violent offender group; CTL, control group) and both tasks across emotions. discrimination. Further, there was a significant main effect of emotion, $F(5.10,188.86)=8.69, p<.001$, and an interaction between emotion and task, $F(4.57,169.12)=6.68, p<.001$, indicating a taskdependent influence of emotional expression on the number of transitions between AOIs. Follow-up ANOVAs showed that there was only a significant effect of emotion for the emotion categorization task, $F(4.37,161.71)=12.40, p<.001$, and not for gender discrimination, $F(5.13,189.87)=1.13, p=.346$. Interestingly, the number of AOI transitions for the different emotion categories during emotion recognition showed a roughly inverted picture of the accuracy data. Accordingly, participants made the most transitions during the attempt to recognize fearful faces and the least transitions for happy faces even though they did not differ significantly from all of the other emotions. Finally, the three-way interaction of group, task and emotion was significant, $F(4.57,169.12)=2.59$, $p<.032$ which was driven only by a group-dependent influence of task for angry faces, $F(1,37)=5.00, p<.032$. When broken down by emotion, follow-up ANOVAs for all other emotions did not yield significant interactions between group and task, all $F<0.52$, all $p>.475$.

It should be noted that the number of AOI transitions showed significant positive associations with self-reported psychopathy measures, i.e., SRP total score, $r=.44, p=.005$ (see Figure 4), and three SRP subscales, i.e., Interpersonal Manipulation, Erratic Lifestyle, and Callous Affect, all $r>.36$, all $p<.026$. For the remaining psychopathy subscale Antisocial Behavior and the reported scores of aggressive behavior, there were no such associations, all $r<.13$, all $p>.441$.

4.3.3 Gaze Transition Entropy. Gaze transition entropy, as developed by Krejtz et al. [2015], was designed to statistically compare fixation transitions between AOIs. With a set of AOIs $\mathcal{S}=\{1, \ldots, s\}$ defined over the stimulus, computation of transition entropy requires construction of first-order transition matrices. Matrix elements contain observed conditional probabilities, from which normalized transition entropy $H_{t}$ is calculated as

$$
H_{t}=-\frac{1}{\log _{2}|\mathcal{S}|} \sum_{i \in \mathcal{S}} p_{i} \sum_{j \in \mathcal{S}} p_{i j} \log _{2} p_{i j}
$$


where $p_{i}$ is the observed (simple) probability of viewing the $i^{\text {th }}$ AOI, $p_{i j}$ is the conditional probability of viewing the $j^{\text {th }}$ AOI given the previous viewing of the $i^{\text {th }}$ AOI, and $|\mathcal{S}|$ is the number of AOIs. Transition entropy $H_{t}$ provides a measure of statistical dependency in the spatial pattern of fixations represented by the transition matrix, and is used to compare one matrix to another.

Weiss et al. [1989] note that a small $H_{t}$ suggests a dependency between fixation points, while a large $H_{t}$ suggests a more random scanning pattern. That is, entropy refers to the "expected surprise" of a given gaze transition. Minimum entropy (0) suggests no expected surprise, indicating that a gaze transition is always expected to the same $j^{\text {th }}$ AOI. Maximum entropy (1, when normalized), on the other hand, suggests maximum surprise, since transition from source $i^{\text {th }}$ AOI to any destination $j^{\text {th }}$ AOI is equally likely. More formally, the term $-p_{i j} \log _{2} p_{i j}$ in (1) reflects the transition's contribution to system entropy, modeled by its probability multiplied by its surprisal [Hume and Mailhot 2013].

Computed transition matrices are shown in Figure 5 and corresponding transition entropies in Figure 6. For transition entropy, there was no significant main effect of group, $F(1,37)=0.01, p=.928$. Only task had a marginally significant effect on transition entropy, $F(1,37)=4.08, p=.051$, showing a tendency for higher entropy during categorization of emotions compared to the gender discrimination (see Figure 6). All remaining main effects and interactions were non-significant, all $F<1.05$, all $p>.391$. Calculations of correlations of transition entropy with self-reported scores of psychopathic and aggressive personality traits did not yield any significant results either, all $r<.28$, all $p>.084$.

\section{DISCUSSION}

The aim of the present study was to investigate different aspects of viewing patterns in male AVOs and matched healthy controls while labeling either gender or emotional expression category of presented faces. AVOs reported significantly higher scores of aggressive behavior and psychopathic traits than control participants. However, groups did not differ in terms of emotion recognition accuracy and there were no general group differences in viewing patterns.

The analysis of dwell time and frequency of the initial fixation on the eye region indicated that healthy controls as well as AVOs exhibited a strong preference to look at the eyes of the displayed faces. Consistent with findings of previous studies, attention to the eyes was influenced by the displayed emotion (e.g., more attention to the eye region for fearful compared to happy faces; [Eisenbarth and Alpers 2011; Scheller et al. 2012; Schurgin et al. 2014]) but also by task demands (e.g., see Smith and Merlusca [2014]). Finally, there were no general group differences and both indices of attention to the eyes were not related to psychopathic traits or aggressive behavior. Thus, our findings yielded no evidence of impaired attentional orienting to the eyes associated with psychopathic traits or in antisocial populations per se.

Including the current work, only three studies investigated scan patterns while viewing faces in antisocial groups, overall showing inconsistent findings [Gillespie et al. 2017; Martin-Key et al. 2017]. Thus, future studies should investigate antisocial individuals classified as psychopaths according to the Psychopathy Checklist
(PCL-R) [Hare 2003], the current gold-standard for the assessment of psychopathy. Further, it remains unclear whether the findings can be generalized to females because most studies only included male participants.

In the second part of the analysis, we examined two additional eye movement parameters in order to explore further possible differences between AVOs and healthy controls with regard to scan patterns, i.e., number of transitions between AOIs and gaze transition entropy. The number of gaze transitions between diagnostic features (operationalized as AOIs) is assumed to be an index for the extent of exploration and was indicated to be related to task difficulty. Accordingly, the number of transitions in the current study was higher for the difficult emotion categorization compared to the easier gender discrimination task. Further, the number of transitions varied across emotions only when they were task-relevant and more difficult emotions such as fear were linked to higher number of AOI transitions than easy emotions such as happiness. Thus, this variable might indicate different levels of task difficulty. Gaze transition entropy was calculated as a measure for the predictability of fixation transitions between AOIs. A trend of higher transition entropy during emotion recognition compared to gender discrimination was consistent with the expectation of less predictable gaze patterns during more complex tasks. According to these parameters, AVOs and healthy controls showed similar exploration behavior. However, higher psychopathic traits were related to higher numbers of transitions between AOIs possibly indicating more rapid switching between diagnostic features of the face in order to collect more information. This association might reflect higher subjective task difficulty associated with higher psychopathic traits. However, this assumption is highly speculative and replication of the current findings is required.

Based on the present results, no final conclusions can be drawn regarding the effects of task or displayed emotion on the presented eye movement variables in the general population. We investigated a specific sample of male individuals with rather low levels of education and thus, our findings might not be applicable to other individuals. Further, future studies should explore face scanning including these innovative gaze analytic methods in order to reexamine our interpretation.

\section{CONCLUSION}

We presented an analysis of viewing patterns while categorizing emotional faces based on standard eye movement parameters such as total dwell time to certain AOIs as well as more innovative gaze analytic metrics (e.g., gaze transition entropy). Here, we investigated viewing patterns of a group of AVOs matched with healthy control participants. According to our analysis, there were no clear differences between groups in terms of attention to the eyes, extent of exploration behavior, or structure of switching patterns between AOIs. The majority of eye tracking studies in clinical psychology does not yet tap the potential of more recently developed analytic methods which could provide additional insight in dysfunctional processes associated with psychological disorders. Therefore, the present study contributes to the implementation of innovative gaze analytic methods and to the development of future standard protocols for eye tracking studies in clinical psychology. 


\section{REFERENCES}

Adolphs, R., Gosselin, F., Buchanan, T. W., Tranel, D., Schyns, P., and Damasio, A. R. (2005). A mechanism for impaired fear recognition after amygdala damage. Nature, 433(7021):68-72.

American Psychiatric Association (2000). Diagnostic and Statistical Manual of Mental Disorders: DSM-IV-TR. Autor, Washington, DC, 4th ed., text rev. edition.

Arizpe, J., Kravitz, D. J., Yovel, G., and Baker, C. I. (2012). Start position strongly influences fixation patterns during face processing: Difficulties with eye movements as a measure of information use. PloS one, 7(2):e31106.

Blair, R. J. (1995). A cognitive developmental approach to morality: Investigating the psychopath. Cognition, 57(1):1-29.

Blair, R. J. (2001). Neurocognitive models of aggression, the antisocial personality disorders, and psychopathy. Journal of Neurology, Neurosurgery \& Psychiatry, 71(6):727-731.

Boll, S. and Gamer, M. (2016). Psychopathic traits affect the visual exploration of facial expressions. Biological psychology, 117:194-201.

Bora, E. and Pantelis, C. (2016). Meta-analysis of social cognition in attentiondeficit/hyperactivity disorder (adhd): Comparison with healthy controls and autistic spectrum disorder. Psychological medicine, 46(4):699-716.

Buss, A. H. and Perry, M. (1992). The aggression questionnaire. Fournal of Personality and Social Psychology, 63(3):452-459.

Chapman, H., Gillespie, S. M., and Mitchell, I. J. (2017). Facial affect processing in incarcerated violent males: A systematic review. Aggression and Violent Behavior

Dadds, M. R., El Masry, Y., Wimalaweera, S., and Guastella, A. J. (2008). Reduced eye gaze explains "fear blindness" in childhood psychopathic traits. Fournal of the American Academy of Child \& Adolescent Psychiatry, 47(4):455-463.

Dadds, M. R., Perry, Y., Hawes, D. J., Merz, S., Riddell, A. C., Haines, D. J., Solak, E., and Abeygunawardane, A. I. (2006). Attention to the eyes and fear-recognition deficits in child psychopathy. The British fournal of Psychiatry, 189(3):280-281.

Dalili, M. N., Penton-Voak, I. S., Harmer, C. J., and Munafò, M. R. (2015). Meta-analysis of emotion recognition deficits in major depressive disorder. Psychological medicine 45(6):1135-1144.

Dawel, A., McKone, E., O'Kearney, R., Sellbom, M., Irons, J., and Palermo, R. (2015) Elevated levels of callous unemotional traits are associated with reduced attentional cueing, with no specificity for fear or eyes. Personality Disorders: Theory, Research, and Treatment, 6(3):216-228

Dawel, A., O'Kearney, R., McKone, E., and Palermo, R. (2012). Not just fear and sadness: Meta-analytic evidence of pervasive emotion recognition deficits for facial and vocal expressions in psychopathy. Neuroscience \& Biobehavioral Reviews, 36(10):22882304

Demenescu, L. R., Kortekaas, R., den Boer, J. A., and Aleman, A. (2010). Impaired attribution of emotion to facial expressions in anxiety and major depression. PloS one, 5(12): 15058.

Duchowski, A. T. (2017). Eye Tracking Methodology: Theory \& Practice. Springer-Verlag, Inc., London, UK, 3rd edition.

Eisenbarth, H. and Alpers, G. W. (2011). Happy mouth and sad eyes: Scanning emotional facial expressions. Emotion, 11(4):860-865.

Ekman, P. (1999). Basic emotions in t. dalgleish and t. power (eds.) the handbook of cognition and emotion pp. 45-60.

Ekman, P. and Friesen, W. V. (1971). Constants across cultures in the face and emotion fournal of Personality and Social Psychology, 17(2):124-129.

Formann, A. K. and Piswanger, K. (1979). Wiener Matrizen-Test (WMT). Beltz Test.

Formann, A. K., Piswanger, K., and Waldherr, K. (2011). Wiener Matrizen-Test 2: Ein Rasch-skaldierter sprachfreier Kurztest zu Erfassung der Intelligenz; Testheft. Hogrefe.

Gamer, M. and Büchel, C. (2009). Amygdala activation predicts gaze toward fearful eyes. Fournal of Neuroscience, 29(28):9123-9126.

Gamer, M., Schmitz, A. K., Tittgemeyer, M., and Schilbach, L. (2013). The human amygdala drives reflexive orienting towards facial features. Current Biology, 23(20):R917R918.

Gillespie, S. M., Rotshtein, P., Beech, A. R., and Mitchell, I. J. (2017). Boldness psychopathic traits predict reduced gaze toward fearful eyes in men with a history of violence. Biological psychology, 128:29-38

Gillespie, S. M., Rotshtein, P., Wells, L. J., Beech, A. R., and Mitchell, I. J. (2015). Psychopathic traits are associated with reduced attention to the eyes of emotional faces among adult male non-offenders. Frontiers in Human Neuroscience, 9:552:1-552:11.

Goeleven, E., De Raedt, R., Leyman, L., and Verschuere, B. (2008). The karolinska directed emotional faces: A validation study. Cognition and Emotion, 22(6):10941118

Gorry, P. A. (1990). General least-squares smoothing and differentiation by the convolution (Savitzky-Golay) method. Analytical Chemistry, 62(6):570-573.

Hare, R. D. (2003). Manual for the hare psychopathy checklist. Revised 2nd ed. Toronto, ON: Multi-Health Systems.

Herzberg, P. Y. (2003). Faktorstruktur, Gütekriterien und Konstruktvalidität der deutschen Uebersetzung des Aggressionsfragebogens von Buss und Perry. Zeitschrift für Differentielle und Diagnostische Psychologie, 24(4):311-323.

Hume, E. and Mailhot, F. (2013). The Role of Entropy and Surprisal in Phonologization and Language Change. In Yu, A. C. L., editor, Origins of Sound Change: Approaches to Phonologization, pages 29-47. Oxford University Press, Oxford, UK.
Jiang, X., Tien, G., Huang, D., Zheng, B., and Atkins, M. S. (2013). Capturing and evaluating blinks from video-based eyetrackers. Behavior Research Methods, 45(3):656-663.

Jones, A. P., Laurens, K. R., Herba, C. M., Barker, G. J., and Viding, E. (2009). Amygdala hypoactivity to fearful faces in boys with conduct problems and callousunemotional traits. American fournal of Psychiatry, 166(1):95-102.

Kennedy, D. P. and Adolphs, R. (2011). Reprint of: Impaired fixation to eyes following amygdala damage arises from abnormal bottom-up attention. Neuropsychologia, 49(4):589-595

Kohler, C. G., Walker, J. B., Martin, E. A., Healey, K. M., and Moberg, P. J. (2009). Facial emotion perception in schizophrenia: A meta-analytic review. Schizophrenia bulletin, 36(5):1009-1019.

Kopp, D., Drenkhahn, K., Dünkel, F., Freyberger, H. J., Spitzer, C., Barnow, S., and Dudeck, M. (2011). Psychische Symptombelastung bei Kurz-und Langzeitgefangenen in Deutschland. Der Nervenarzt, 82(7):880-885.

Krejtz, K., Duchowski, A., Szmidt, T., Krejtz, I., González Perilli, F., Pires, A., Vilaro, A., and Villalobos, N. (2015). Gaze transition entropy. ACM Transactions on Applied Perception, 13(1):4:1-4:20.

Lozier, L. M., Cardinale, E. M., VanMeter, J. W., and Marsh, A. A. (2014). Mediation of the relationship between callous-unemotional traits and proactive aggression by amygdala response to fear among children with conduct problems. JAMA Psychiatry, 71(6):627-636.

Lundqvist, D., Flykt, A., and Öhman, A. (1998). The Karolinska Directed Emotional Faces (KDEF). CD ROM from Department of Clinical Neuroscience, Psychology section, Karolinska Institutet.

Marsh, A. A. and Blair, R. J. R. (2008). Deficits in facial affect recognition among antisocial populations: A meta-analysis. Neuroscience \& Biobehavioral Reviews, 32(3):454-465.

Marsh, A. A., Finger, E. C., Mitchell, D. G., Reid, M. E., Sims, C., Kosson, D. S., Towbin, K. E., Leibenluft, E., Pine, D. S., and Blair, R. J. (2008). Reduced amygdala response to fearful expressions in children and adolescents with callous-unemotional traits and disruptive behavior disorders. American fournal of Psychiatry, 165(6):712-720.

Martin-Key, N. A., Graf, E. W., Adams, W. J., and Fairchild, G. (2017). Facial emotion recognition and eye movement behaviour in conduct disorder. Fournal of Child Psychology and Psychiatry.

Morris, J. S., Frith, C. D., Perrett, D. I., Rowland, D., Young, A. W., Calder, A. J., and Dolan, R. J. (1996). A differential neural response in the human amygdala to fearful and happy facial expressions. Nature, 383(6603):812-815.

Nummenmaa, L., Hyönä, J., and Calvo, M. G. (2006). Eye movement assessment of selective attentional capture by emotional pictures. Emotion, 6(2):257-268.

Paulhus, D. L., Neumann, C. S., and Hare, R. D. (2009). Manual for the Self-Report Psychopathy scale. Toronto: Multi-health systems.

R Core Team (2016). R: A Language and Environment for Statistical Computing. R Foundation for Statistical Computing, Vienna, Austria.

Savitzky, A. and Golay, M. J. E. (1964). Smoothing and differentiation of data by simplified least squares procedures. Analytical Chemistry, 36(8):1627-1639.

Scheller, E., Büchel, C., and Gamer, M. (2012). Diagnostic features of emotional expressions are processed preferentially. PloS one, 7(7):e41792.

Schurgin, M., Nelson, J., Iida, S., Ohira, H., Chiao, J., and Franconeri, S. (2014). Eye movements during emotion recognition in faces. Fournal of vision, 14(13):14:1-14:16.

Schyns, P. G., Bonnar, L., and Gosselin, F. (2002). Show me the features! Understanding recognition from the use of visual information. Psychological Science, 13(5):402-409.

Sheehan, D. V., Janavs, J., Harnett-Sheehan, K., Sheehan, M., Gray, C., Lecrubier, Y., Weiller, E., Hergueta, T., Allgulander, C., Kadri, N., Baldwin, D., and Even, C. (2010). M.I.N.I.: Mini International Neuropsychiatric Interview, German Translation Version 6.0.0 (DSM-IV)

Siegenthaler, E., Costela, F. M., McCamy, M. B., Di Stasi, L. L., Otero-Millan, J., Sonderegger, A., Groner, R., Macknik, S., and Martinez-Conde, S. (2014). Task difficulty in mental arithmetic affects microsaccadic rates and magnitudes. European fournal of Neuroscience, 39(1):1-8.

Smith, M. L., Cottrell, G. W., Gosselin, F., and Schyns, P. G. (2005). Transmitting and decoding facial expressions. Psychological Science, 16(3):184-189.

Smith, M. L. and Merlusca, C. (2014). How task shapes the use of information during facial expression categorizations. Emotion, 14(3):478-487.

SR Research (2008). EyeLink User Manual. SR Research Ltd., 5516 Main St., Osgoode, ON, Canada K0A 2W0. Version 1.4.0.

Tracy, J. L. and Robins, R. W. (2008). The automaticity of emotion recognition. Emotion, $8(1): 81-95$

Uljarevic, M. and Hamilton, A. (2013). Recognition of emotions in autism: A formal meta-analysis. Fournal of Autism and Developmental Disorders, 43(7):1517-1526.

Weiss, R. S., Remington, R., and Ellis, S. R. (1989). Sampling distributions of the entropy in visual scanning. Behavior Research Methods, Instruments, \& Computers (BRMIC), 21(3):348-352.

Whalen, P. J., Kagan, J., Cook, R. G., Davis, F. C., Kim, H., Polis, S., McLaren, D. G, Somerville, L. H., McLean, A. A., Maxwell, J. S., et al. (2004). Human amygdala responsivity to masked fearful eye whites. Science, 306(5704):2061-2061.

Wilson, K., Juodis, M., and Porter, S. (2011). Fear and loathing in psychopaths: A meta-analytic investigation of the facial affect recognition deficit. Criminal fustice and Behavior, 38(7):659-668 